\section{Mieloma múltiple en Chile: pasado, presente y futuro del programa nacional de drogas antineoplásicas (PANDA). Revisión de 1.103 pacientes}

\author{
CAMILA PEÑA ${ }^{1, a}$, CHRISTINE ROJAS ${ }^{2}$, HERNÁN ROJAS $^{3}$, \\ PABLO SOTO $^{4}$, DANIELA CARDEMIL ${ }^{5}$, SANDRA ARANDA $^{6}$, \\ CAROLINA CONTRERAS ${ }^{7}$, GABRIEL LA ROCA $^{8}$, MOISÉS RUSSO ${ }^{9,10}$, \\ CLAUDIO PÉREZ ${ }^{11}$, VIVIANNE LOIS ${ }^{12, a}$
}

\section{Survival of 1,103 Chilean patients with multiple myeloma receiving different therapeutic protocols from 2000 to 2016}

Background: Multiple myeloma (MM) is one of the most common malignancies found in hematology. Aim: To describe the features of patients with MM and perform a survival analysis according to the different treatment protocols used between 2000 and 2016. Material and Methods: Analysis of the database of the Chilean national anti-neoplastic drug program. Information was obtained from 1,103 patients, with a median age of 64.5 years (range 27-95) and a male to female ratio of 1:1.2. Results: The mean overall survival (OS) of patients receiving or not receiving Thalidomide was 46 and 30 months, respectively $(p<0.01)$. The mean OS of patients treated before 2007 (treated with melphalan and prednisone) and between 2007 and 2012 (treated with thalidomide and dexamethasone) was 36 and 48 months respectively. In the group starting in 2013 (treated with cyclophosphamide, thalidomide and dexamethasone) the median survival had not been reached at 20 months of follow up ( $p=0.01$ for all comparisons). Autologous transplantation (AT) was carried out in only $18 \%$ of the eligible patients. The median OS of the patients who receive an AT had not been reached at 48 month compared with 36 month among those who did not received the procedure $(p<0.01)$. Conclusions: Even though overall survival has improved with time, new drugs must be introduced in our protocols to obtain similar results to those obtained worldwide.

(Rev Med Chile 2018; 146: 869-875)

Key words: Clinical Protocols; Cyclophosphamide; Multiple Myeloma; Thalidomide; Transplantation, Autologous.

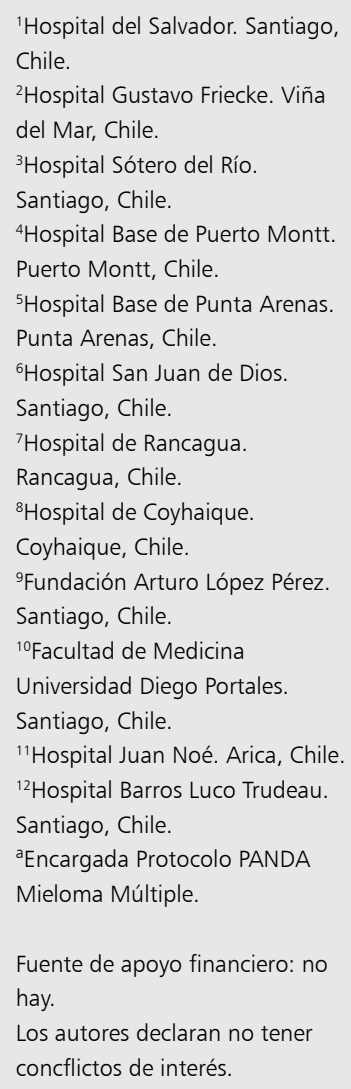

$\mathrm{E}$ 1 mieloma múltiple (MM) es una neoplasia de células plasmáticas que se caracteriza por secreción de una proteína monoclonal y síntomas como anemia, hipercalcemia, falla renal y lesiones óseas. Clásicamente, se describe como $1 \%$ de las neoplasias y 10 a $15 \%$ de las neoplasias hematológicas $^{1-3}$. El Ministerio de Salud chileno estimó su incidencia en la década pasada (entre 2003 y 2007) en 3,3/100.000 habitantes, con un estimado de 330 casos nuevos al año. Se cree, sin embargo, que esta incidencia ha aumentado. El MM es, por tanto, una de las neoplasias más frecuentes en hematología.

Existe escasa información sobre su epidemio- 
logía y clínica en Latinoamérica. Recientemente se publicó un interesante reporte de 852 pacientes en el que participaron 23 instituciones de Argentina, Perú, Chile, México y Brasil ${ }^{4}$, un gran esfuerzo de Hungria et al en esta materia.

En nuestro país, la revisión más importante en cuanto a clínica y epidemiología es una de 245 pacientes de Conte et al. de $2007^{5}$. Existe además otro reporte de 50 pacientes del Hospital Naval de Valparaíso ${ }^{6}$. También es posible encontrar mayor información sobre tratamiento y sobrevida en $\mathrm{MM}$, especialmente de parte de los grupos que realizan trasplante en nuestro país ${ }^{7-9}$.

El Programa Nacional de Drogas Antineoplásicas (PANDA) es una entidad que forma parte del Ministerio de Salud, y es el encargado de crear los protocolos de diagnóstico, tratamiento y seguimiento de las neoplasias hematológicas del sistema público chileno. Además, realiza la revisión y actualización continua de los protocolos y realiza en ellos los cambios que sean pertinentes.

Los resultados de los distintos protocolos PANDA de MM no han sido revisados, por lo que decidimos caracterizar a nuestros pacientes y realizar un análisis de sobrevida de ellos.

El objetivo del presente estudio es caracterizar a los pacientes con diagnóstico de $\mathrm{MM}$ en el sistema público chileno y evaluar su sobrevida con los diferentes protocolos utilizados.

\section{Material y Métodos}

El presente es un estudio retrospectivo, multicéntrico, de diferentes centros PANDA en Chile. Se recopilaron los datos desde el año 2000 hasta el año 2016 de una base de datos ministerial.

\section{Caracterización de los pacientes}

Se buscó los datos epidemiológicos y de laboratorio de la base de datos ministerial y se solicitó la información faltante a cada centro. Se realizó tabla ad hoc. Se midió específicamente edad, sexo, índice pronóstico de acuerdo al International Staging System (ISS) que clasifica según albúmina y beta-2-microglobulina, tipo de MM y tratamiento utilizado.

\section{Protocolos de tratamiento}

En pacientes candidatos a trasplante autólogo de progenitores hematopoyéticos (TAPH) y sobrevida alcanzada en cada uno, hasta el 2006 se usó como inducción los protocolos con melfalán y prednisona (MP) y vincristina, doxorrubicina y dexametasona (VAD).

En 2007 se incorporó al arsenal terapéutico la talidomida. El protocolo cambió a talidomida y dexametasona (taldex) en candidatos a trasplante autólogo de progenitores hematopoyéticos (TAPH).

En el 2013 se cambió el régimen de primera línea a ciclofosfamida, talidomida y dexametasona (CTD). Actualmente no existe financiamiento en el sistema público para drogas como bortezomib o lenalidomida

En los pacientes no candidatos a TAPH, el manejo se basa en melfalán, prednisona, talidomida (MPT).

Para el estudio de sobrevida se evaluó estos 3 diferentes protocolos en pacientes candidatos a TAPH.

\section{Trasplante autólogo}

Desde el 2010 se realiza en la Unidad Hematología Intensiva del Hospital del Salvador quimioterapia en altas dosis y rescate con trasplante autólogo. La indicación para TAPH definida en el PANDA incluye pacientes menores de 60 años, sin comorbilidades importantes, que logran remisión completa (RC) o muy buena remisión parcial (MBRP) con el tratamiento de inducción. Se comparó la sobrevida de los pacientes en que se realizó TAPH con aquellos que no calificaron para el procedimiento.

\section{Estadística}

Se realizó estadística descriptiva. El análisis de sobrevida se realizó mediante el método de Kaplan-Meier. Comparaciones de sobrevida entre grupos se realizaron mediante el método de log-rank. Todos los análisis fueron realizados en STATA 12.

\section{Resultados}

\section{Características clínicas}

Se obtuvo datos de 1.103 pacientes. La mediana de edad fue de 64,5 años, con rango entre 27 y 95 años. La relación Hombre:Mujer fue de 1:1,2. Los pacientes menores de 60 años correspondieron a $36 \%$. En cuanto a tipos de MM, se obtuvo la información en 894 pacientes (81\%). Se observó 
Tabla 1. Generalidades de los pacientes

\begin{tabular}{|llcl|}
\hline & & $\mathbf{n}$ & $\mathbf{\%}$ \\
Género & Femenino & 603 & 55 \\
1.103 ptes & Masculino & 500 & 45 \\
Edad & $<$ o $=60$ años & 393 & 36 \\
1.103 ptes & $>60$ años & 710 & 64 \\
Etapa ISS & ISS I & 156 & 21 \\
751 ptes & ISS II & 238 & 32 \\
& ISS III & 357 & 47 \\
Tipo & IgG & 539 & 60 \\
896 ptes & IgA & 244 & 27 \\
& $\mathrm{CL}$ & 83 & 9 \\
& IgD & 4 & 0,6 \\
& IgE & 1 & 0,6 \\
& IgM & 10 & 1 \\
& No secretor & 13 & 1 \\
& Biclonal & 2 & 0,6 \\
\hline
\end{tabular}

IgG en $60 \%$, IgA en $27 \%$ y MM de cadenas livianas (CL) en $9 \%$. El pronóstico por ISS se obtuvo en 751 pacientes $(68 \%)$. Se diagnosticó en ISS de I a $21 \%$, en ISS II a $32 \%$ y $47 \%$ en ISS III (Tabla 1 ).

\section{Sobrevida}

\section{Según ISS}

Con seguimiento medio de 101 meses, la sobrevida media de los pacientes diagnosticados en ISS de I fue de 66 meses, la de los pacientes en ISS de II fue de 44 meses y los con ISS de III de 28 meses. La sobrevida global (SG) (Figura 1A) a 5 y 10 años de pacientes en ISS de I fue de $54 \%$ y $36 \%$, respectivamente; de los en ISS de II fue de $37 \%$ y $26 \%$, respectivamente; y de los en ISS3 de $27 \%$ y $21 \%$, respectivamente $(\mathrm{p}<0,001)$.

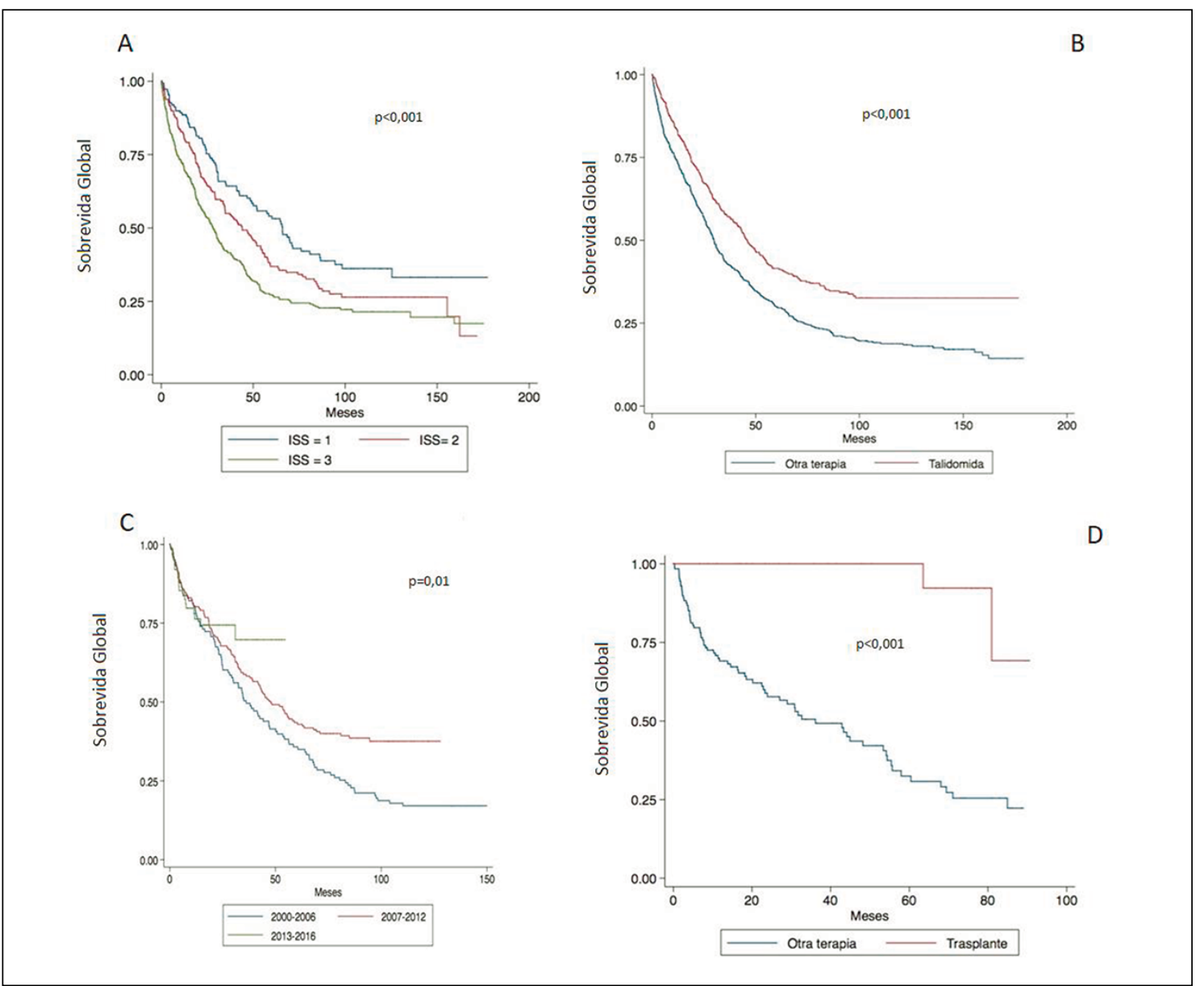

Figura 1. A: Sobrevida global según ISS; B: Sobrevida global según si se usó talidomida o no; C: Sobrevida global en menores de 60 años según los diferentes protocolos usados. D: Sobrevida global según si se realizó trasplante o no, en pacientes candidatos. 


\section{Según uso de drogas nuevas (talidomida)}

Con un seguimiento medio de 122 meses en los pacientes que no recibieron talidomida y de 88 meses en los pacientes que sí la recibieron, la sobrevida media del primer grupo fue de 30 meses versus 46 meses del segundo ( $\mathrm{p}<0,001)$. La SG a 5 y 10 años del grupo sin talidomida fue de 30 y $19 \%$, respectivamente. En el grupo de pacientes que recibió talidomida fue de 41 y $33 \%$, respectivamente (Figura 1B).

\section{Según período estudiado}

El seguimiento medio del grupo de pacientes diagnosticados previo a 2007 fue de 146 meses, del grupo entre 2007 y 2012 fue de 98 meses y del grupo posterior a 2012 de 20 meses. La sobrevida media del primer grupo fue de 36 meses, la del segundo grupo fue de 48 meses y la de los pacientes diagnosticados desde el 2013 aún no ha sido alcanzada $(\mathrm{p}=0,01)$.

La SG a 3, 5 y 10 años en el primer período fue de 50,36 y $17 \%$, respectivamente, en el segundo período fue de 59,44 y $38 \%$, respectivamente; y en el tercer período fue de $70 \%$ a 3 años (Figura 1C).

\section{Según realización de TAPH}

Desde el 2010 se realizó TAPH en 18\% de los pacientes candidatos, $6,6 \%$ del total de pacientes. La mediana de sobrevida de los pacientes sin TAPH fue de 36 meses versus no alcanzada aún a los 48 meses en los que sí se realizó el procedimiento $(\mathrm{p}<0,001)$. La SG a 3 y 5 años de los pacientes sin TAPH fue de 51 y $33 \%$, respectivamente, versus $100 \%$ a 5 años de los trasplantados (Figura 1D).

\section{Discusión}

Reunimos 1.103 casos, el mayor grupo estudiado hasta la fecha en Chile. La edad promedio fue de 64,5 años, similar a lo descrito internacionalmente de, aproximadamente, 65 años. La edad media observada en la cohorte latinoamericana fue de 60 años ${ }^{9}$, lo que podría mostrar una diferencia con los estudios europeos o norteamericanos, en que el MM se presenta en pacientes mayores.

Llamó la atención que no observamos un predominio de sexo masculino, a diferencia de lo descrito internacionalmente ${ }^{10}$. Este fenómeno ya había sido reportado antes en Chile por Conte et $\mathrm{al}^{4} \mathrm{y}$ por Minnicelli et $\mathrm{al}^{11}$, en una cohorte brasileña de bajo nivel social. La razón requiere mayor estudio, sin embargo, podría deberse a que los pacientes latinoamericanos de sexo femenino (particularmente las de menos recursos) hacen mayor uso del sistema de atención de salud.

En cuanto al tipo de MM, encontramos un bajo número de MM de CL ( solo 9\%), similar resultado a la anterior cohorte chilena ${ }^{4}$. Esto no se reprodujo en el estudio latinoamericano ${ }^{9}$, que reportó $16 \%$. No tenemos clara la causa de este subdiagnóstico en nuestro país, pero es posible que se trate de la interpretación errónea de la electroforesis de proteínas por parte de la comunidad médica, en el sentido de que este tipo de MM se presenta generalmente con hipogammaglobulinemia sin un componente monoclonal. También se observó menor frecuencia de MM IgD, E y biclonales, los que se describen internacionalmente en $2 \%$, aproximadamente, cada uno ${ }^{10,12,13}$. En cuanto al subdiagnóstico de MM IgM, esto puede explicarse por la confusión que a veces se presenta en diferenciar MM IgM y macroglobulinemia de Waldenström en médicos no especialistas ${ }^{14}$.

El ISS solo se obtuvo en $68 \%$ de los pacientes. Esto se debe a que en años anteriores el índice pronóstico se medía por Durie Salmon ${ }^{15}$, que no incluye beta 2 microglobulina. Es importante mencionar que algunos centros PANDA no cuentan con este examen y deben solicitarlo al extrasistema, lo que se traduce, lamentablemente, en esta falta de etapificación. Sin perjuicio de lo anterior, los resultados en cuanto a sobrevida según ISS I, II o III resultaron muy similares a lo descrito por Greip en $2005^{16}$, diferenciándose claramente las 3 curvas, validando este score en nuestro país y corroborando los datos de Conte et al publicados en $2008^{17}$. Sin embargo, a diferencia de estos estudios, encontramos un mayor número de pacientes en etapas más avanzadas, coincidiendo con una cohorte del sistema público mexicano ${ }^{18}$ y una brasileñ ${ }^{19}$, pudiendo explicarse por el menor acceso precoz al sistema de salud en estos pacientes.

No se realiza hibridación fluorescente in situ (FISH) de rutina, por lo que no se obtuvo datos sobre el nuevo score (R-ISS) sugerido por el International Myeloma Working Group (IMWG) ${ }^{20}$.

Previo a las nuevas drogas se usó por muchos años MP y luego VAD para candidatos a trasplante. Con estos regímenes de tratamiento, la sobrevida no aumentó significativamente y solo 
se lograba RC en 5\% de ellos ${ }^{21}$. En Chile, los datos publicados anteriormente no arrojaron mejores resultados, con una sobrevida media de 33 meses y una SG a 5 años de $23 \%$, con una alta mortalidad los primeros 6 meses $^{4}$. Nuestros resultados lo confirman, con una sobrevida media de 30 meses en los pacientes no tratados con drogas nuevas (la mayoría previo al 2007).

Afortunadamente, la introducción de las llamadas "nuevas drogas" (talidomida, bortezomib y lenalidomida) ha aumentado progresivamente la sobrevida de estos pacientes. Esto se refleja en los resultados publicados por la Clínica Mayo, en donde la sobrevida media previa al 2001 fue de 2,5 años, entre 2001 y 2005 de 4,6 años, y entre 2006 y 2010 de 6,1 años ${ }^{22,23}$.

Debido al alto costo de las demás drogas, la talidomida fue la droga incluída en el protocolo PANDA desde el 2007. Esta droga demostró tener buen perfil de seguridad, no provocar mielotoxicidad ${ }^{24}$ y mejorar sobrevida libre de progresión y SG en múltiples estudios ${ }^{25,26}$. No sorprende, entonces, el aumento en la sobrevida media observada por nosotros con su introducción en todos los grupos etarios.

En un comienzo, el régimen elegido fue taldex, basado en los buenos resultados obtenidos en ese entonces para candidatos a TAPH ${ }^{27-29}$. De hecho, en los servicios públicos de Latinoamérica, el taldex es uno de los regímenes más usados?.

En 2013, luego de revisar la literatura, se eligió el CTD como régimen de inducción en pacientes candidatos a TAPH, un régimen relativamente barato, eficaz y administrado por vía oral en la mayoría de los casos. Morgan et $\mathrm{al}^{30}$ demostraron que el CTD es un régimen seguro, con baja tasa de citopenias, buena tasa de respuesta $(82,5 \%)$ y mayores respuestas completas que el régimen convencional. Otros estudios mostraron resultados similares $^{31,32}$. En la región, Brasil tiene reportes con este régimen como inducción previo a trasplante ${ }^{33}$, en donde se vio al menos muy buena respuesta parcial en $61 \%$ de los pacientes, con una SG a 5 años estimada de 55\%. Nuestros resultados son similares, aunque aún con menor seguimiento.

Sorprendentemente, los diferentes protocolos para candidatos a TAPH estudiados en nuestro medio mostraron diferencia significativa entre si en cuanto a SG en los 3 períodos. Aparte de la introducción de la talidomida, creemos que la mejor sobrevida del tercer protocolo (CTD) puede deberse al mejor manejo de soporte que se les ofrece a estos pacientes actualmente, por ejemplo, en tratamientos antibióticos, mayor ingreso a unidades de pacientes críticos, acceso a hemodiálisis de agudo, etc.

En relación a TAPH, solo $18 \%$ de los candidatos se trasplantó, una cifra francamente inferior a lo deseable. La proporción de trasplante de la cohorte latinoamericana fue de 58\% de los candidatos, muy superior a nuestro resultado. Creemos que se puede inferir que ese $18 \%$ es aproximadamente el porcentaje de RC + MBRP con los actuales protocolos basados en talidomida. Esto nos pone en alerta, pues esa proporción de respuestas es bastante inferior a lo reportado con otras drogas como bortezomib o lenalidomida, que describen respuesta a RC + MBRP de hasta $70 \%{ }^{34-36}$.

La sobrevida de los pacientes que lograron llegar a TAPH fue muy favorable, lo que se condice con algunos estudios internacionales, aunque debe destacarse que estos han mostrado resultados disímiles $^{37,38}$. Sin embargo, nuestros resultados deben analizarse con cautela, ya que en nuestro medio solo llegan a TAPH los mejores candidatos, es decir, los más jóvenes y en RC o MBRP.

Sabemos que nuestros resultados distan de lo reportado internacionalmente, ya que el acceso a drogas más potentes y a TAPH es limitado. Esta realidad es preocupante, especialmente por la brecha que se observa con el sistema privado. Lamentablemente, este hecho de repite en la región. Recientemente, Tarín-Arzaga et al. reportaron 2 grupos de pacientes con MM tratados en México, uno del sistema privado y otro del sistema público $^{18}$. Demostraron que la diferencia de tratamiento otorgado en ambos sistemas influye significativamente en las respuestas y sobrevida de los pacientes.

Es importante recalcar que el presente estudio no es un estudio poblacional, por lo que puede haber sesgo en los datos obtenidos. Los centros enviaron información a veces incompleta, y no se obtuvieron los datos de todos los centros PANDA.

Sin embargo, esta es la mayor publicación de pacientes con MM en Chile. Creemos que es un gran aporte para evaluar los avances logrados y nuestras falencias. Una de las tareas prioritarias debe ser que los centros tratantes tengan las herramientas diagnósticas y pronósticas indispensables. Nuestro gran objetivo para el futuro debe ser mejorar los regímenes de inducción de nuestros 
pacientes, especialmente con la introducción de drogas más potentes en cuanto a respuestas, y así aumentar nuestra cobertura en TAPH.

\section{Referencias}

1. Siegel RL, Miller KD, Jemal A. Cancer Statistics, 2017. CA Cancer J Clin 2017; 67 (1): 7-30.

2. Bladé J, Cibeira MT, Fernández de Larrea C, Rosiñol L. Multiple myeloma. Ann Oncol 2010; 21 Suppl 7: vii3139.

3. Dimopoulos MA, Terpos E. Multiple myeloma. Ann Oncol 2010; 21 Suppl 7: vii143-50.

4. Hungria VT, Maiolino A, Martínez G, Duarte GO, Bittencourt R, Peters L, et al. Observational study of multiple myeloma in Latin America. Ann Hematol 2017; 96 (1): 65-72.

5. Conte G, Figueroa G, Lois V, Cabrera ME, León A, García H, et al. Mieloma múltiple en Chile. Características clínicas y sobrevida. Rev Med Chile 2007; 135: 1111-7.

6. Barría K, Maldonado J, Álvarez R, Rodríguez M, Cortés M. Casuística de gammapatía monoclonal de significado incierto y mieloma múltiple en el Hospital Naval Almirante Nef, Viña del Mar, Chile. Rev Med Chile 2010; 138 (6): 788-90.

7. Flores C, Conte G, Fardella P, Araos D, Alfaro J, Aravena $\mathrm{P}$, et al. [Autologous transplant (AT) with peripheral-blood stem-cell rescue for multiple myeloma]. Rev Med Chile 2005; 133 (8): 887-93.

8. Puga B, Molina J, Andrade A, Guerra C, Ardila A, Alvarez G. Primeros diez trasplantes autólogos de progenitores hematopoyéticos en adultos, en el servicio público de salud en Chile. Rev Med Chile 2012; 140 (9): 1207-12.

9. Sarmiento M, Lira P, Ocqueteau M, Rodríguez MA, García MJ, Jara V, et al. Experiencia de 22 años de trasplante autólogo de células hematopoyéticas en pacientes con mieloma múltiple o amiloidosis sistémica. 19922014. Rev Med Chile 2014; 142: 1497-501.

10. Kyle RA, Gertz MA, Witzig TE, Lust JA, Lacy MQ, Dispenzieri A, et al. Review of 1027 patients with newly diagnosed multiple myeloma. Mayo Clin Proc 2003; 78 (1): 21-33.

11. Minnicelli C, Maciel JF, Hassan R, Lemos TM. Clinical and epidemiological features of multiple myeloma patients from a low socio-economic region of Brazil. Rev Bras Hematol Hemoter 2015; 37 (5): 354-5.

12. Kuliszkiewicz-Janus M, Zimny A, Sokolska V, Sasiadek M, Kuliczkowski K. Immunoglobulin D myeloma-problems with diagnosing and staging (own experience and literature review). Leuk Lymphoma 2005; 46: 1029-37.

13. Pisani F, Petrucci MT, Giannarelli D, Bongarzoni V, Montanaro M, De Stefano V, et al. IgD multiple myeloma a descriptive report of 17 cases: survival and response to therapy. J Exp Clin Cancer Res 2012; 31: 17.

14. Schuster S, Rajkumar V, Dispenzieri A, Morice W, Moreno A, Ansell S, et al. IgM multiple myeloma: Disease definition, prognosis, and differentiation from Waldenstrom's macroglobulinemia Am J Hematol 2010; 85: 853-5.

15. Durie B, Salmon S. A clinical staging system for multiple myeloma: Correlation of measured myeloma cell mass with presenting clinical features, response to treatment, and survival. Cancer 1975; 36: 842-54.

16. Greipp P, San Miguel J, Durie B, Crowley J, Barlogie B, Bladé J, et al. International staging system for multiple myeloma. J Clin Oncol 2005; 23: 3412-20.

17. Conté LG, Figueroa MG, Lois VV, Cabrera CME, León RA, García LH, et al. Valor pronóstico del nuevo sistema de etapificación internacional en mieloma múltiple. Comparación con el sistema de Durie-Salmon. Rev Med Chile 2008; 136 (1): 7-12.

18. Tarín-Arzaga L, Arredondo-Campos D, Martínez-Pacheco V, Martínez-González O, Ramírez-López A, Gómez-De León A, et al. Impact of the affordability of novel agents in patients with multiple myeloma: Real-world data of current clinical practice in Mexico. Cancer. 2018 Feb 20.

19. Hungria VT, Maiolino A, Martínez G, Colleoni GW, Coelho EO, Rocha L, et al. Confirmation of the utility of the International Staging System and identification of a unique pattern of disease in Brazilian patients with multiple myeloma. Haematologica 2008; 93: 791-2.

20. Palumbo A, Avet-Loiseau H, Oliva S, Lokhorst HM, Goldschmidt H, Rosinol L, et al. Revised International Staging System for Multiple Myeloma: A Report From International Myeloma Working Group. J Clin Oncol 2015; 33 (26): 2863-9.

21. Barlogie B, Shaughnessy J, Tricot G, Jacobson J, Zangari M, Anaissie E, et al. Treatment of multiple myeloma. Blood 2004; 103: 20-32.

22. Rajkumar SV. Multiple Myeloma: 2014 update on diagnosis, risk-stratification, and management. Am J Hematol 2014; 89: 998-1009.

23. Kumar SK, Dispenzieri A, Lacy MQ, Gertz MA, Buadi FK, Pandey S, et al. Continued improvement in survival in multiple myeloma: changes in early mortality and outcomes in older patients. Leukemia 2014; 28 : 1122-8.

24. Zervas K, Mihou D, Katodritou E, Pouli A, Mitsouli CH, Anagnostopoulos A, et al. VAD-doxil versus VAD-doxil 
plus thalidomide as initial treatment for multiple myeloma: results of a multicenter randomized trial of the Greek Myeloma Study Group. Ann Oncol 2007; 18 (8): 1369-75.

25. Lokhorst HM, Schmidt-Wolf I, Sonneveld P, van der Holt B, Martin H, Barge R, et al. Thalidomide in induction treatment increases the very good partial response rate before and after high-dose therapy in previously untreated multiple myeloma. Haematologica 2008; 93 (1): 124-7.

26. Barlogie B, Tricot G, Anaissie E, Shaughnessy J, Rasmussen E, van Rhee F, et al. Thalidomide and hematopoietic-cell transplantation for multiple myeloma. N Engl J Med 2006; 354 (10): 1021-30.

27. Rajkumar SV, Blood E, Vesole D, Fonseca R, Greipp PR; Eastern Cooperative Oncology Group. Phase III clinical trial of thalidomide plus dexamethasone compared with dexamethasone alone in newly diagnosed multiple myeloma: a clinical trial coordinated by the Eastern Cooperative Oncology Group. J Clin Oncol 2006; 24 (3): 431-6.

28. Rajkumar SV, Rosiñol L, Hussein M, Catalano J, Jedrzejczak W, Lucy L, et al. Multicenter, randomized, double-blind, placebo-controlled study of thalidomide plus dexamethasone compared with dexamethasone as initial therapy for newly diagnosed multiple myeloma. J Clin Oncol 2008; 26 (13): 2171-7.

29. Vogl DT, Liu SV, Chong EA, Luger SM, Porter DL, Schuster SJ, et al. Post-transplant outcomes of induction therapy for myeloma: thalidomide and dexamethasone versus doxorubicin, vincristine, and dexamethasone prior to high-dose melphalan with autologous stem cell support. Am J Hematol 2007; 82 (12): 1071-5.

30. Morgan GJ, Davies FE, Gregory WM, Bell SE, Szubert AJ, Navarro Coy N, et al. Cyclophosphamide, thalidomide, and dexamethasone as induction therapy for newly diagnosed multiple myeloma patients destined for autologous stem-cell transplantation: MRC Myeloma IX randomized trial results. Haematologica 2012; 97 (3): 442-50.

31. Wu P, Davies FE, Horton C, Jenner MW, Krishnan B,
Alvares CL, et al. The combination of cyclophosphamide, thalidomide and dexamethasone is an effective alternative to cyclophosphamide-vincristine-doxorubicin-methylprednisolone as induction chemotherapy prior to autologous transplantation for multiple myeloma: a case-matched analysis. Leuk Lymphoma 2006; 47 (11): 2335-8.

32. Sidra G, Williams CD, Russell NH, Zaman S, Myers B, Byrne JL. Combination chemotherapy with cyclophosphamide, thalidomide and dexamethasone for patients with refractory, newly diagnosed or relapsed myeloma. Haematologica 2006; 91 (6): 862-3.

33. Crusoe Ede Q, Higashi F, Padilha MP, Miranda EC, Quero AA, Almeida M de S, et al. Outcomes of autologous transplantation for multiple myeloma according to different induction regimens. Rev Bras Hematol Hemoter 2014; 36 (1): 19-24.

34. Rajkumar V. Doublets, triplets, or quadruplets of novel agents in newly diagnosed myeloma? Hematology Am Soc Hematol Educ Program 2012; 2012: 354-61.

35. Landau H, Giralt H. Treatment of Transplant-Eligible Patients with Multiple Myeloma in 2014. Hematol Oncol Clin N Am 2014; 28: 815-27.

36. Reeder CB, Reece DE, Kukreti V, Chen C, Trudel S, Hentz J, et al. Cyclophosphamide, bortezomib and dexamethasone (CyBorD) induction for newly diagnosed multiple myeloma: High response rates in a phase II clinical trial. Leukemia 2009; 23 (7): 1337-41.

37. Attal M, Harousseau JL, Stoppa AM, Sotto JJ, Fuzibet JG, Rossi JF, et al. A prospective, randomized trial of autologous bone marrow transplantation and chemotherapy in multiple myeloma. N Engl J Med 1996; 335: 91-7.

38. Child JA, Morgan GJ, Davies FE, Owen RG, Bell SE, Hawkins K, et al. High-dose chemotherapy with hematopoietic stem-cell rescue for multiple myeloma. $\mathrm{N} \mathrm{Engl}$ J Med 2003; 348: 1875-83.

39. Palumbo A, Bringhen S, Petrucci MT, Musto P, Rossini F, Nunzi M, et al. Intermediate-dose melphalan improves survival of myeloma patients aged 50 to 70 : results of a randomized controlled trial. Blood 2004; 104: 3052-7. 